\title{
Surgical Options for Transposition of the Great Arteries With Ventricular Septal Defect and Left Ventricular Outflow Tract Obstruction: Evolution and Functional Impact
}

\author{
Alsayed Salem, MD, PhD \\ National Heart Institute, Cairo, Egypt
}

\section{ABSTRACT}

Surgery for D-transposition of the great arteries, ventricular septal defect and left ventricular outflow tract obstruction has continuously evolved to achieve optimal hemodynamic performance across the right and left ventricular outflow tracts, include predominantly native tissues, and preserve pulmonary valve function. Classically, three types of repair are applied: Rastelli, REV, and translocation procedures. The concept of translocation remains more radical and exposed to many modifications. Its extensive reconstructive nature extends its application to similar lesions with discordant ventriculo-arterial connection. We tried to compare the values and limitations of these surgical options, emphasizing how a more anatomical repair could impact the functional outcome.

\section{INTRODUCTION}

With discordant ventriculo-arterial connection and biventricular circulation, attempts are always made to restore the systemic ventricle to the aorta and the pulmonary ventricle to the pulmonary artery connection without mixing and with the least possible degree of outflow obstruction and/or regurgitation. The most frequently encountered examples are D-transposition of great arteries with ventricular septal defect and pulmonary stenosis (D- TGA, VSD, PS) or (TGA lll).

Repair strategies and techniques for TGA III went through a long way of evolution and received to- many modifications. Three types of repair were reported: Rastelli repair [Rastelli 1969], reparation letage ventriculaire (REV) [Lecompte 1982], and Nikaidoh operation (aortic translocation) [Nikaidoh 1984]. The Rastelli concept of repair remains nearly constant over the years. The REV operation underwent only one major modification, pulmonary root translocation [Da Silva 2000], while the Nikaidoh operation underwent several modifications that started with insertion of the right ventricle-pulmonary artery (RV-PA) conduit then the more interestingly half-turned truncal switch (or truncal rotation) [Yamagishi 2003] and double root translocation [Hu 2007].

Received Fuly 17, 2020; accepted August 26, 2020.

Correspondence: Alsayed Salem, National Heart Institute, Cairo, Egypt; +201112319555 (e-mail: sayedmmsalem@gmail.com).
Although relieving left ventricular outflow tract obstruction (LVOTO) is an essential part of all types of repair, the discordant ventriculo-arterial connection creates a necessity for dealing with both outflow tracts. Morever, the mode of RV-PA connection remains far from the perfect solution and considered as the main reason for multiple reoperations [Kreutzer 2000; Dearani 2001; Horer 2007].

When the left ventricle (LV) is re-routed to the aorta, the normal right ventricular outflow tract (RVOT) is used as a new LVOT and the future RV-PA junction is transferred to the anterior right ventricular wall. The new RVOT might be a prosthetic conduit [Rastelli 1969] or native pulmonary artery - either valve-less due to sacrification of the stenotic valvular component [Lecompte 1982; Nikaidoh 1984] with valve preservation, at least partially, when the pulmonary root is translocated [Da Silva 2000]. The stenosed sub-pulmonary component remains left sided and excluded because the VSD is used as LVOT [Rastelli 1969; Lecompte 1982].

When the aorta is translocated posteriorly over the reconstructed LVOT, the RVOT is kept as a base for the new connection preserving the right ventricle (RV) volume and allowing an orthotopic origin of the translocated PA (with or without valve preservation) or its prosthetic substitute [Nikaidoh 1984; Da Silva 2000; Yamagishi 2003; Hu 2007].

So, in our opinion, evolution of surgery for D-TGA, VSD, PS and similar lesions could be described, more appropriately, according to the concept of each repair strategy rather than the anatomical and technical details focusing on the functional outcome and flow dynamics across the two outflow tracts. It depends again on the way of biventricular outflow tract reconstruction and the mode of RV-PA connection.

A - Biventricular outflow tract reconstruction:

Transformation from re-routing to translocation was the major evolution involving the intra-cardiac part of repair, and although it involves mainly the way of LVOT reconstruction, it affects the RVOT in the same degree of importance.

1 - Re-routing: (heterotopic origin of both great arteries) - LVOT: tunneled LV to aorta using the VSD as outflow

- RVOT: anterior origin of PA from anterior RV wall 2 - Translocation: (orthotopic origin of both great arteries)

- LVOT: transferred aorta to LV with VSD closure

- RVOT: basal origin of PA from RVOT 
B - RV-PA connection:

1 - Tissue of origin of pulmonary valve and artery:

- Completely non autogenous; in Rastelli repair

- Completely or mainly autogenous; in REV and pulmonary translocation

2 - Native pulmonary valve function:

- Absent valve function; REV and original Nikaidoh operation

- Complete or partial valve function; pulmonary trans-

location with or without trans-annular patch.

Two main observations could be reported following this description:

1 - There were three major evolutions; one involved the way of outflow tract reconstruction and tow involved the mode of RV to PA connection.

a - First, the transformation from re-routing to translocation with its hemodynamic advantages and the reducing of reoperation rate for LVOTO. REV is similar to Rastelli operation at this point, both are re-routing types of repair, in spite of some technical variations especially the more aggressive resection of the outlet septum. But the shape of the LVOT remains nearly the same, due to the fare distance between the aortic root and the LVOT. Morever, the takeoff of the neo pulmonary outflow remains anterior (heterotopic) in a re-routing strategy and basal (orthotopic) in posterior aortic translocation. $\mathrm{b}$ - Second, the transmission from using completely non autogenous tissues for RVOT reconstruction in Rastelli repair to at least partial incorporation of the native pulmonary tissue into the posterior wall preserving the potential for growth. Preserving autogenous pulmonary tissue was an advantage of REV, Nikaidoh operation and pulmonary translocation. So, regarding the tissue of origin of RV-PA connection; REV operation was similar in the general concept to the Nikaidoh operation and away from the Rastelli repair.

c - Third, the preservation of native valve function, at least partially. Insisting on preserving native valve function, together with growth potential, of course, probably came later as a result of lessons learned from the deleterious effects of chronic pulmonary regurgitation post trans-annular patch repair of TOF. Pulmonary root translocation was the first and important step in this respect. Initially, it was associated with re-routing and considered as the major modification of REV operation but later on it was associated with aortic translocation in the form of double root translocation or truncal rotation. So, regarding the preservation of native valve function at RV-PA junction, it was an advantage of modifications that came many years later in contrary to both totally valve-less repair of the REV and Nikaidoh operations or prosthetic valved conduits of Rastelli repair.

2 - Each type of repair underwent some technical modifications that did not result in major functional changes but just were variations on how to apply the major concepts of repair. Prominent examples are resection of the conal septum during Rastelli repair and the different ways of handling the coronary arteries during aortic translocation.
Technical details and evaluation of the outcome of different types of repair were discussed generously elsewhere. However, functional values and limitations for each type of repair could be summarized before trying to reach a road map for decision making.

The most classic repair:

Rastelli operation remained the classic repair for TGA, VSD and PS with good short and midterm results and two main advantages: technical feasibility and immediate excellent valve function. Both allowed the risk of surgery to be very low. Rastelli operation was considered to be more suitable to: 1) adult patients and older children, 2) large right ventricular cavity, 3) extreme double outlet right ventricle (DORV), PS, sub-aortic VSD when translocation is not possible and TGA, VSD and pulmonary atresia when translocation has no value [Hu 2008].

However, the long-term outcome is unfavorable with high late mortality and reoperation rate, due to deterioration of the hemodynamic performance of both the LT and RT outflow tracts [Kreutzer 2000; Dearani 2001; Horer 2007]. Rastelli repair also is limited in small infants due to the rarity of small size conduits and rapid deterioration of its function. Many anatomical situations are not suitable to this repair, small or remote VSD, straddling atrio-ventricular valves (AVVs) and small size RV [Hu 2008].

More extensive resection of the conal septum and widening of the VSD antero-superiorly assumed to reduce the reoperation rate for LVOTO after Rastelli repair similar to what occurs with REV operation but should be done selectively as a part of any corrective surgery using the VSD as an outflow of the LV. Morever, routine enlargement of the VSD may increase the incidence of heart block up to $11 \%$ compared with only $2 \%$ for REV operation when resection is limited mainly to the conal septum and around 3\% when antero-superior enlargement of VSD is done selectively during Rastelli repair [Alsoufi 2009].

The most feasible repair:

The major advantages of REV technique over Rastelli operation are absence of the need for prosthetic conduit, less $\mathrm{RV}$ volume reduction, and more straight connection of the $\mathrm{LV}$ to the aorta as the outlet septum is sub totally resected as an intimate part of the procedure [Mosca 2015; Honjo 2013], however, this does not lead to a complete freedom from reoperation for LVOTO [Hazekamp 2010]. These advantages together with noticeable technical feasibility makes REV operation unable to gain prevalence [Mosca 2015; Hazekamp 2010; Vouhé 2014].

As no conduit is required, REV operation is more suitable to small infants than Rastelli repair at the expense of higher early mortality due pulmonary regurgitation as a sequelae [Alsoufi 2009; Honjo 2013]. Also, it is more applicable to smaller babies than translocation, which is more technically difficult before the age of six months [Hazekamp 2010]. REV, like Rastelli, remains a re-routing strategy more suitable to patients with at least good RV cavity size and diagnosis of DORV, PS and sub-aortic VSD and with similar anatomical limitations, small or remote VSD, straddling AVVs and small size RV [Alsoufi 2009; Honjo 2013; Hazekamp 2010]. 
Functionally, single pulmonary root translocation could be considered as the major modification of REV operation with the added benefit of preserving pulmonary valve function, at least partially [Da Silva 2000]. Importantly, it was noticed that many patients had a near normal pulmonary valve once explanted and freed from the sub-valvular muscular and fibrous tissues, despite having a preoperative high left ventricular-pulmonary arterial gradient [Da Silva 2009; Da Silva 2012]. But it retains the same anatomical limitations of REV and remains of no value if the pulmonary valve is atretic or severely hypo plastic [Da Silva 2012]. In contrary, we considered Metras modification with aortic autograft extension of the PA and avoidance of Lecompte maneuver [Metras 1997] as a good technical modification, but it did not change the main concept of REV or alter the functional outcome.

The most radical repair:

Translocation might be single aortic, single pulmonary or combined translocation. Aortic translocation results in a more anatomical repair regarding the flow dynamics though the outflow tracts [Mosca 2015; Honjo 2013; Hazekamp 2010; Hazekamp 2007], and pulmonary translocation preserves the potential for growth and saves acceptable valve function when totally preserved or completed anteriorly with mono-cusp [Yamagishi 2003; Hu 2007].

Aortic translocation is the essential component to apply the concept of radical biventricular outflow tract reconstruction leading to orthotopic position of the aorta and pulmonary artery or its substitute [Nikaidoh 1984; Hazekamp 2007]. According to the associated mode of RV-PA connection, the result will be one of the following alternatives [Nikaidoh 1984; Yamagishi 2003; Hu 2007; Hazekamp 2007]:

1 - Aortic translocation plus valve-less translocation of the pulmonary artery to the RVOT similar to extra cardiac part of REV repair.

2 - Aortic translocation plus prosthetic RV- PA conduit similar to extra cardiac part of Rastelli repair.

3 - Combined aortic and pulmonary root translocation either separate or as enblock truncal rotation.

Combining aortic and pulmonary root translocation as applied by Yamagishi and Hu collects most of the advantages, including better flow dynamics and preserved native valve tissue and function at the expense of some technical complexity [Yamagishi 2003; Hu 2008; Hazekamp 2007].

Value of translocation: [Mosca 2015; Honjo 2013; Hazekamp 2007; Furlanetto 2013; Lim 2005; Furlanetto 2016]

1 - Superior hemodynamics across LT and RT outflow tracts, due to better alignment with the great arteries. The LVOT is wide and straight, and systemic blood is not passing through the VSD or the intraventricular tunnel.

2 - RV function expected to be better, due to preserved pulmonary valve function and no anterior ventriculotomy. Morever, less RV volume reduction as a result of straight VSD patch.

3 - It allows autogenous RV-PA connection with excellent growth potential.

4 - It might be the only possible corrective surgery in cases of remote VSD, small RV size, AVV straddling precluding complete septation through the classic intra-cardiac re-routing.
5 - Allows functionally accepted surgical correction in the absence of valved conduits.

6 - The concept of translocation extended to include other indications like D-TGA/IVS with PS and L-TGA with PS. Furthermore, translocation applied by Furlanito for repair of D-TGA without PS and for Tausig-Bing anomaly as a more definitive repair rather than the arterial switch operation (ASO) in which arteries are switched but roots are not and considered as incomplete definitive repair.

Limitations: [Mosca 2015; Honjo 2013; Vouhé 2014; Metras 1997]

1 - More complex and technically demanding repair with much longer aortic cross-clamp time.

2 - It risks the integrity of the aortic root and coronary arteries.

3 - Major coronary artery abnormalities preclude the translocation technique especially the anterior looping of the right coronary artery (RCA), which makes mobilization of the aortic root difficult and also poses a risk of significant tension on the RCA.

4 - Minimal benefit when the aorta is translocated over a very limited distance in case of very small pulmonary valve and LVOT.

Surgical options and decision making:

Historically, several surgical options were offered for management of TGA III:

1 - Arterial switch operation (ASO), VSD closure, and LVOTO resection/pulmonary valve repair.

2 - Rastelli operation is the classic repair.

3 - REV operation with its major modification pulmonary translocation.

4 - Nikaidoh operation with its major modifications double root translocation and half turned truncal switch operation.

5 - Atrial switch procedures, VSD closure and LVOT resection with or without a conduit.

6 - Uni-ventricular repair is reserved for few indications.

ASO with VSD closure and LVOTO resection/pulmonary valve repair is the best option, and if possible, should be considered first. For TGA/IVS/ LVOTO, the initial atrial switch procedures, combined with VSD closure and LVOT resection, with or without a conduit, proved unsatisfactory in the long term and largely have been abandoned [Mosca 2015]. Uni-ventricular repair is reserved for few indications if $\mathrm{RV}$ is hypoplastic or small and remote VSD requiring complex intra-cardiac tunnel [Honjo 2013; Hazekamp 2010].

So, if we excluded atrial switch and uni-ventricular repair, the following steps are usually followed:

a - The anatomy of LVOTO is the first element to be analyzed. The anatomy of the sub-valvar obstruction must be clarified whether it can be relieved or not. The native pulmonary valve is evaluated to determine whether it is subnormal but could serve as aortic valve and allowing ASO or abnormal but good enough to serve as the pulmonary valve if translocated. It might be severely dysplastic and not usable [Vouhé 2014].

$\mathrm{b}$ - If ASO is not possible due to complex LVOTO, the choice is mostly made between Rastelli, REV, and translocation, considering the value and limitations of each repair. 
In this regard, apart from surgeon preference and institutional experiences, the choice is influenced by the age and size of the patient, ventricular function and size and anatomical details, including spatial relationship of great vessels, position and size of the VSD, anatomy of the conal septum, presence and severity of straddling of AVV and anatomy of the coronary arteries [Mosca 2015; Al-Jughiman 2015].

\section{CONCLUSION}

Improving long-term outcome and reducing the need for multiple reoperations require optimal reconstruction of both the RT and LT outflow tracts and avoidance of prosthetic conduits together with preserving native valve function. On the other side, decreasing the risk of surgery is a permanent goal that surgeons always try to achieve through simplifying the technically demanding and complex procedures. Achieving these two targets requires balance between surgical skills, team experience, and patient characteristics.

The concept of translocation remains more radical and carries higher potential for evolution and wider spectrum of indications. Its functional superiority might be achieved at the expense of noticeable technical complexity and longer cross-clamp time in comparison with other types of repair. Gain is not without risk, which should be calculated. All surgical options must be taken into consideration and selecting the best surgical option is not only a matter of surgeon choice but also patient choice.

\section{REFERENCES}

Al-Jughiman MK, Al-Omair MA, Van Arsdell GS, et al. 2015. D-Transposition of the great arteries with ventricular septal defect and left ventricular outflow tract obstruction (D-TGA/VSD/LVOTO): A survey of perceptions, preferences, and experience. Pediatr Cardiol 36:896-905.

Alsoufi B, Awan A, Al-Omrani A, et al. 2009. The Rastelli procedure for transposition of the great arteries: Resection of the infundibular septum diminishes recurrent left ventricular outflow tract obstruction risk. Ann Thorac Surg 88: 137-143.

Da Silva JP, Baumgratz JF, Da Fonseca L. 2000. Pulmonary Root Translocation in transposition of great arteries repair. Ann Thorac Surg 69:643-645

Da Silva JP, da Fonseca L. 2009. Pulmonary root translocation. Optechstcvs 23-34.

Da Silva JP, da Fonseca L, Lopes LM, et al. 2012. Pulmonary root translocation in malposition of great arteries repair allows right ventricular outflow tract growth. J Thorac Cardiovasc Surg 143:1292-1298.

Dearani JA, Danielson GK, Puga FJ, et al. 2001. Late results of the Rastelli operation for transposition of the great arteries. Semin Thorac Cardiovasc Surg Pediatr Card Surg Annu. 4:3-15.

Furlanetto G, Furlanetto BHS, Henriques S, et al. 2013. Double root switch: A complete anatomical correction for transposition of the great arteries. World J Pediatr Congenit Heart Surg. 4(1):98-102.
Furlanetto G, Furlanetto BHS, Teixeira EM, et al. 2016. Double root switch repair for taussig-bing anomaly. World J Pediatr Congenit Heart Sur. 7(3):367-371.

Hazekamp M, Gomez AA, Koolbergen DR, et al. 2010. Surgery for transposition of the great arteries, ventricular septal defect and left ventricular outflow tract obstruction: European Congenital Heart Surgeons Association multicenter study. Eur J Cardiothorac Surg 38: 699—706.

Hazekamp M, Portela F, Bartelings M. 2007. The optimal procedure for the great arteries and left ventricular outflow tract obstruction. An anatomical study. Eur J Cardiothorac Surg 31:879-887.

Honjo O, Kotani Y, Bharucha T, et al. 2013. Anatomical factors determining surgical decision-making in patients with transposition of the great arteries with left ventricular outflow tract obstruction. Eur J Cardiothorac Surg 44:1085-1094.

Horer J, Schreiber C, Dworak E, et al. 2007. Long-term results after the Rastelli repair for transposition of the great arteries. Ann Thorac Surg 83:2169-75.

Hu S, Li S, Wang X, et al. 2007. Pulmonary and aortic root translocation in the management of transposition of the great arteries with ventricular septal defect and left ventricular outflow tract obstruction. J Thorac Cardiovasc Surg 133:1090-1092.

Hu S, Liu Z, Li S, et al. 2008. Strategy for biventricular outflow tract reconstruction: Rastelli, REV, or Nikaidoh procedure? J Thorac Cardiovasc Surg 135:331-338.

Kreutzer C, De Vive J, Oppido G, et al. 2000. Twenty-five-year experience with Rastelli repair for transposition of the great arteries. J Thorac Cardiovasc Surg. 120:211-23.

Lecompte Y, Neveux JY, Leca F, Zannini L, et al. 1982. Reconstruction of the pulmonary outflow tract without prosthetic conduit. J Thorac Cardiovasc Surg 84:727-733.

Lim HG, Lee JR, Bae, EJ, et al. 2005. Pulmonary Root Translocation for Repair of Taussig-Bing Anomaly with Interrupted Arch. Ann Thorac Surg 80:1943-1945.

Metras D, Kreitmann B, Riberi A, et al. 1997. Extending the concept of the autograft for complete repair of transposition of the great arteries with ventricular septal defect and left ventricular outflow tract obstruction: A report of ten cases of a modified procedure. J Thorac Cardiovasc Surg 114: 746-754.

Mosca RS. 2015. TGA, VSD, LVOTO: evolution of surgical therapy. J Thorac Cardiovasc Surg 149:1356-1357.

Nikaidoh H. 1984. Aortic translocation and biventricular outflow tract reconstruction: a new surgical repair for transposition of the great arteries associated with a ventricular septal defect and pulmonary stenosis. J Thorac Cardiovasc Surg 88:365-372.

Rastelli GC, Wallace RB, Ongley PA. 1969. Complete repair of transposition of the great arteries with pulmonary stenosis: a review and report of a case corrected by using a new surgical technique. Circulation 39:83-95.

Vouhé PR. 2014. Transposition of the great arteries with ventricular septal defect and left ventricular outflow tract obstruction: how to choose in the surgical armamentarium? Eur J Cardiothorac Surg 45: 935-936.

Yamagishi M, Shuntoh K, Matsushita T, et al. 2003. Half-turned truncal switch operation for complete transposition of the great arteries with ventricular septal defect and pulmonary stenosis. J Thorac Cardiovasc Surg 125:966-968. 